\title{
Stargazing or scientific yardstick? The future of speckle tracking echocardiography in the management of ascending aortic aneurysm
}

\author{
Patrick T. Roughneen, MD, Ghannam Al-Dossari, MD, and Abe DeAnda, Jr, MD
}

\footnotetext{
From the Division of Cardiothoracic Surgery, Department of Surgery, University of Texas Medical Branch Galveston, Galveston, Tex.

Disclosures: Authors have nothing to disclose with regard to commercial support.

Received for publication Nov 22, 2016; accepted for publication Dec 1, 2016; available ahead of print Jan 10 , 2017.

Address for reprints: Abe DeAnda, Jr, MD, UTMB-Galveston, Division of Cardiothoracic Surgery, 301 University Blvd. Galveston, TX 77555 (E-mail: abdeanda@UTMB.edu).

J Thorac Cardiovasc Surg 2017;153:779-80 $0022-5223 / \$ 36.00$

Copyright (c) 2016 by The American Association for Thoracic Surgery http://dx.doi.org/10.1016/j.jtcvs.2016.12.001
}

Management of an enlarged ascending aorta is guided by a number of factors enumerated by consensus statements that are updated regularly. Although definitive guidelines exist, ${ }^{1}$ there is a grey zone for those aortas that are not quite large enough to operate on and that may or may not enlarge over time. Having an objective and predictive method of assessing the mechanical properties of the in situ aorta would aid in predicting which aortas might eventually need intervention.

In this issue of the Journal, Alreshidan and colleagues ${ }^{2}$ present a novel approach to the monitoring of patients with aneurysmal disease of the ascending aorta. This preliminary work suggests that assessment of an in vivo stiffness index, as calculated by speckle tracking echocardiography (STE), correlates with estimates of ex vivo stiffness of the excised aorta in patients with aneurysmal disease requiring surgery. This early work clearly has merit and if further validated could be considered for broader clinical application.

However, several practical issues need to be discussed and considered for STE to be embraced by aortic surgeons for its widespread clinical application. The most obvious issue is that the current static dimensional assessment of the ascending aorta by computed tomography (CT) or magnetic resonance imaging angiography is a simple, easy, and reproducible method of determining the need for surgical therapy or monitoring with a relatively simple set of guidelines. The introduction of STE in the assessment of such patients would provide a more physiologic yardstick that assesses the biological characteristics of the aortic tissue as a diagnostic and predictive tool, but with some obvious practical disadvantages. By the authors' own recognition, the aorta becomes stiffer with patient aging. It also becomes stiffer the further one progresses from the aortic valve distally. There is also regional variability noted because circumferential segments of aneurysmal aorta resected at an identical plane all have different stiffness

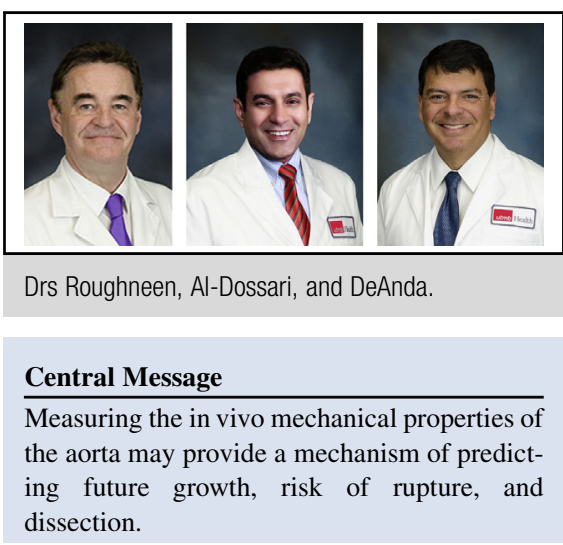

See Article page 781 .

characteristics. Last, a certain aortic stiffness may have different predictive values for patients with different aortic pathology, such as Marfan disease or Ehlers-Danlos syndrome, or for the disease processes of dissection and aneurysmal dilatation as separate pathologic entities. Given this, it is clear that guidelines or clinical algorithms for patients with ascending aortic aneurysms could become extremely complex if STE becomes a mainstream technology.

The clinical ease with which STE can be implemented also requires further discussion. As noted, the current assessment of the ascending aorta uses CT angiography or magnetic resonance imaging, which are both less invasive than transesophageal echocardiography, the current diagnostic tool in STE. If STE were to prove itself a more predictive yardstick, the invasiveness of transesophageal echocardiography would still be an inhibitory factor in the widespread and routine adoption of STE. This would beg the question as to whether technologic improvements in transthoracic echocardiography, as a less-invasive tool, could be adopted in STE.

The work of Alreshidan and colleagues ${ }^{2}$ is an important contribution to scientific literature in the field of aortic disease. The concept that assessment of the biomechanical behavior of the aorta is a primary physiologic yardstick we should evaluate in aortic aneurysmal disease certainly has theoretic merit. The provisional data presented support scientific validity of STE, but further studies are required to investigate and develop the application of such technology. 
By assuming validation, the predictive value of such technology in determining who is at risk for the progression of aneurysmal disease or dissection processes will represent a significant advancement in our understanding and management of aortic disease. The apparent inhibitions to such application as discussed do not prohibit the future significant clinical application of STE, but may rather identify its use as a hybrid technology in conjunction with CT angiography and magnetic resonance angiography. It is likely that STE will find its niche as a dynamic physiologic tool to further identify and manage the high-risk aorta, so why not shoot for the stars?

\section{References}

1. Hiratzka LF, Bakris GL, Beckman JA, Bersin RM, Carr VF, Casey DE Jr, et al. 2010 ACCF/AHA/AATS/ACR/ASA/SCA/SCAI/SIR/STS/SVM Guidelines for the diagnosis and management of patients with thoracic aortic disease: a report of the American College of Cardiology Foundation/American Heart Association Task Force on Practice Guidelines, American Association for Thoracic Surgery, American College of Radiology, American Stroke Association, Society of Cardiovascular Anesthesiologists, Society for Cardiovascular Angiography and Interventions, Society of Interventional Radiology, Society of Thoracic Surgeons, and Society for Vascular Medicine (developed in collaboration with the American College of Emergency Physicians). J Am Coll Cardiol. 2010;55:e27-129.

2. Alreshidan M, Shahmansouri N, Chung J, Lash V, Emmott A, Leask RL, et al. Obtaining the biomechanical behavior of ascending aortic aneurysm by using novel speckle tracking echocardiography. J Thorac Cardiovasc Surg. 2017;153: $781-8$. 